\title{
A rare pulmonary zygomycosis manifested as chronic mediastinitis and bronchial perforation
}

\section{To the Editors:}

Zygomycosis is an emerging infection caused by fungi of class Zygomycetes, order Mucorales. It occurs mostly in diabetic and immunocompromised patients, but occasionally occurs in immunocompetent individuals [1]. After rhinocerebral zygomycosis, pulmonary zygomycosis, which carries a high mortality rate, is the second most common presentation [1]. Common manifestations of pulmonary zygomycosis mainly include different types of pulmonary infiltrates [2], and other rare manifestations such as endobronchial mass lesions or mediastinitis. However, in the literature, bronchial perforation caused by zygomycetes has not been previously documented. This report presents the case of a diabetic patient infected with Rhizopus sp., which resulted in chronic mediastinitis and bronchial perforation.

A 76-yr-old, Chinese, never-smoking female with uncontrolled type 2 diabetes experienced a productive cough for 6 months prior to admission. This patient denied having fever, breathlessness, night sweating or body weight loss. Physical examination revealed a moderately nourished female without respiratory distress or fever. Upon admission, laboratory studies revealed a haemoglobin level of $10.5 \mathrm{~g} \cdot \mathrm{dL}^{-1}$, white blood cell count $5.4 \times 10^{3}$ cells per $\mu \mathrm{L}$ (53.3\% neutrophils, $28.3 \%$ lymphocytes, $6 \%$ monocytes and $12 \%$ eosinophils), platelet count $355 \times 10^{3}$ cells per $\mu \mathrm{L}$, creatinine $0.9 \mathrm{mg} \cdot \mathrm{dL}^{-1}$, C-reactive protein $1.7 \mathrm{mg} \cdot \mathrm{dL}^{-1}$, fasting glucose level $311 \mathrm{mg} \cdot \mathrm{dL}^{-1}$ and glycated haemoglobin $14.7 \%$. The chest radiograph on admission revealed partial atelectasis of the right lung lower lobe and a right perihilar mass. Computed tomography $(\mathrm{CT})$ of the chest detected a $5.7 \times 5.5 \times 3.7-\mathrm{cm}$ mass lesion on the right hilum (fig. 1a) and multiple lymphadenopathies on the paratracheal area. Fibreoptic bronchoscopy examination further revealed a distinct bronchial perforation on the right main bronchus and whitish caseous tissue in the mediastinum (fig. 1b). The narrowed right intermediate bronchus was also compressed by the mediastinal lesion. Several biopsies were obtained from the mediastinal caseous tissue. No acid-fast bacilli were identified in the biopsy specimens by acid-fast staining and no Mycobacterium tuberculosis grew after prolonged culture. A PCR for mycobacteria complex in the biopsy specimen yielded a negative result. However, many broad ribbon-like hyphae were identified microscopically in biopsy specimens using Gram staining and acid-fast staining. Periodic acid-Schiff and GrocottGomori methenamine silver stains further confirmed the hyphae within the necrotic tissue (fig. 1d). Rhizopus sp., which belongs to one genus of zygomycetes, grew from the specimen cultured on potato dextrose agar (BD BBL ${ }^{\mathrm{TM}}$; BD, Taipei, Taiwan). Slide culture mounts of the isolate stained with lactophenol cotton blue revealed typical morphologic features of Rhizopus sp., with the presence of rhizoids directly below the sporangiophores, unbranched sporangiophores, inconspicuous apophysis, globose sporangia and oval sporangiospores. The diagnosis of mediastinal zygomycosis in the patient was based on histological findings and cultures of the causal agent.
Conventional amphotericin B (AMB) was administered intravenously at a dosage of $1 \mathrm{mg} \cdot \mathrm{kg}^{-1} \cdot \mathrm{day}^{-1}$ for 34 days because the patient refused surgical intervention. Simultaneously, control of hyperglycaemia was accomplished with insulin administration. The patient displayed significant clinical and radiological improvement with conventional AMB treatment. AMB treatment was changed to liposomal AMB (L-AMB; $3 \mathrm{mg} \cdot \mathrm{kg}^{-1} \cdot \mathrm{day}^{-1}$ ) due to the development of an acute kidney injury. When L-AMB was administered for 4 weeks, repeated chest CT established a continued decrease in size of the mediastinal mass from $5.5 \times 3.7 \times 5.7 \mathrm{~cm}$ to $2.4 \times 1.4 \times 2.3 \mathrm{~cm}$ (fig. $1 \mathrm{c}$ ). After a cumulative dose of $6,300 \mathrm{mg}$ L-AMB was administered for 6 weeks, the treatment of the patient was accomplished. Then, the patient was discharged in a stable condition. 2 months after discharge, the patient had no recurrent symptoms. 1 yr after discharge, the patient was quite well and the follow-up chest CT showed a complete resolution of the mediastinal lesion.

Chronic mediastinitis, also known as sclerosing, fibrosing, or granulomatous mediastinitis, is rare and most often caused by Histoplasma capsulatum or tuberculosis infection. The symptoms of chronic mediastinitis are always nonspecific, and may be associated with the involvement of the bronchus, superior vena cava (SVC), oesophagus, pulmonary vessels and mediastinal nerves. Bronchial perforation is also a rare complication of mediastinal tuberculosis and histoplasmosis [3]. Bronchial perforation is caused by necrotising granulomatous lymphadenitis that causes extrusion of calcified lymph nodes into the bronchial lumen.

Quite a few characteristics of this case differ from four previous reports of patients with mediastinal zygomycosis [4-7]. In this case, mediastinal zygomycosis had airway involvement manifested as bronchial perforation and a compressed bronchus, compared with SVC involvement caused by mediastinal zygomycosis in two previous reports $[4,5]$. Uncontrolled diabetes was the predisposing factor for mediastinal zygomycosis in this case, but a case with leukaemia and three others without definite predisposing factors were described in previous reports [4-7].

It is interesting to discuss the pathogenic mechanisms behind the rare clinical presentation in this case. The typical route for zygomycetes to induce pulmonary disease was thought to be through spore inhalation [2]. There have been reports that zygomycosis could present as endobronchial mass lesions [8]. First, inhaled spores may germinate in the bronchus and form an endobronchial lesion. At this point, endobronchial zygomycetes may directly invade and erode the bronchial wall into the adjacent mediastinum. However, bronchoscopy did not recognise any obvious endobronchial mass lesions in this case. Secondly, the possible mechanism is analogous to the pathogenesis observed in mediastinal tuberculosis and histoplasmosis. Inhaled spores are initially transported to the mediastinal lymph nodes by alveolar macrophages. Spores germinate into hyphal elements and induce granulomatous mediastinitis 

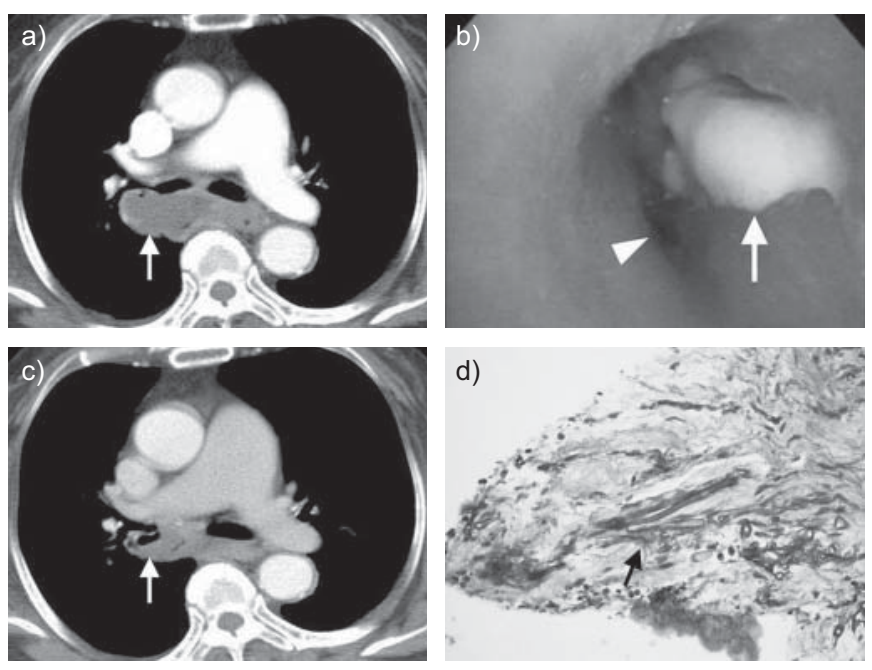

FIGURE 1. Mediastinal zygomycosis in a 76-yr-old diabetic female. a) The initial chest computed tomography (CT) scan showed a $5.7 \times 5.5 \times 3.7-\mathrm{cm}$ mass lesion with air density on the right hilum (arrow). b) Fibreoptic bronchoscopy revealed a wedge-shaped perforation on the membranous portion of the right main bronchus and whitish caseous tissue in the mediastinum (arrow). It was also noted that the narrowed intermediate bronchus was compressed by the mediastinal lesion (arrowhead). c) After antifungal treatment for 2 months, repeated CT revealed a decrease in the size of the mediastinal mass (arrow). d) Periodic acid-Schiff-stained mediastinal tissue section showed broad, pauciseptate hyphae with branches at right angles (arrow) within necrotic tissue. Magnification: 200x.

because macrophages in diabetic hosts are impaired in the suppression of spore germination [9]. Because zygomycosis has the characteristic features of angioinvasion, vascular thrombosis and tissue necrosis [10], mediastinal zygomycosis may erode into the adjacent bronchus and produce a bronchial perforation. Though the second mechanism is a more reasonable explanation for the presentation in this case, further studies are still needed for confirmation.

Based on an early diagnosis and reversal of the underlying predisposing condition, this patient with subacute presentation of mediastinal zygomycosis was treated successfully with $\mathrm{AMB}$ and L-AMB. Along with histoplasmosis and tuberculosis, zygomycosis should be considered in patients with clinical manifestations of chronic mediastinitis and bronchial perforations.
H-C. Liu*\#, M-S. Jan ", Y-C. Lin", W-L. Lin", T-C. Wu* C-N. Huang ${ }^{\S}, \mathrm{C}-\mathrm{M}$. Chen ${ }^{\#}$ and M-C. Lu ${ }^{\top f}$

*Division of Chest Medicine, ${ }^{5}$ Division of Endocrinology and Metabolism, ${ }^{f}$ Division of Infectious Diseases, Dept of Internal Medicine, Chung Shan Medical University Hospital, "Dept of Microbiology and Immunology, Chung Shan Medical University, "Dept of Life Sciences, National Chung-Hsing University, and 'Dept of pathology, Chung Shan Medical University Hospital, Taichung, Taiwan.

Correspondence: M-C. Lu, Dept of Internal Medicine, ChungShan Medical University Hospital, No. 110, Section 1, Jian-Guo North Road, Taichung, Taiwan. E-mail: yokoki2@gmail.com

Statement of Interest: None declared.

\section{REFERENCES}

1 Roden M, Zaoutis $\mathrm{T}$, Buchanan $\mathrm{W}$, et al. Epidemiology and outcome of zygomycosis: a review of 929 reported cases. Clin Infect Dis 2005; 41: 634-653.

2 Lee FY, Mossad SB, Adal KA. Pulmonary mucormycosis: the last 30 years. Arch Intern Med 1999; 159: 1301-1309.

3 Seo JB, Song KS, Lee JS, et al. Broncholithiasis: review of the causes with radiologic-pathologic correlation. Radiographics 2002; 22: S199-S213.

4 Marwaha RK, Banerjee AK, Thapa BR, et al. Mediastinal zygomycosis. Postgrad Med J 1985; 61: 733-735.

5 Robertson B, Bautista M, Russell T, et al. Fibrosing mediastinitis secondary to zygomycosis in a twenty-two-month-old child. Pediatr Infect Dis J 2002; 21: 441-442.

6 Connor B, Anderson R, Smith J. Mucor mediastinitis. Chest 1979; 75: 525-526.

7 Leong AS. Granulomatous mediastinitis due to Rhizopus species. Am J Clin Pathol 1978; 70: 103-107.

8 Husari AW, Jensen WA, Kirsch CM, et al. Pulmonary mucormycosis presenting as an endobronchial lesion. Chest 1994; 106: 18891891.

9 Levitz SM, Selsted ME, Ganz T, et al. In vitro killing of spores and hyphae of Aspergillus fumigatus and Rhizopus oryzae by rabbit neutrophil cationic peptides and bronchoalveolar macrophages. J Infect Dis 1986; 154: 483-489.

10 Ribes JA, Vanover-Sams CL, Baker DJ. Zygomycetes in human disease. Clin Microbiol Rev 2000; 13: 236-301.

DOI: $10.1183 / 09031936.00155110$

\section{Pulmonary endarterectomy in sickle cell haemoglobin C disease}

\section{To the Editors:}

Pulmonary hypertension $(\mathrm{PH})$ is a common complication of sickle cell disease (SCD), with a prevalence of $10-20 \%$, and is often a cause of death in this patient population [1]. PH can be related to two different mechanisms: 1) chronic haemolysis with endothelial dysfunction, reduction in nitric oxide bioavailability and subsequent proliferative vasculopathy; or 2) vaso-occlusive complications resulting from erythrocyte sickling and hyperviscosity [2]. According to a recent report, up to $23 \%$ of SCD patients with $\mathrm{PH}$ have evidence of perfusion 